\title{
Formal Modelling for Ada Implementations: Tasking Event-B
}

\author{
A. Edmunds ${ }^{1}$, A. Rezazadeh and M.J. Butler \\ ${ }^{1}$ ae2@ecs.soton.ac.uk \\ Department of Electronics and Computer Science \\ University of Southampton
}

\begin{abstract}
This paper describes a formal modelling approach, where Ada code is automatically generated from the modelling artefacts. We introduce an implementation-level specification, Tasking Event-B, which is an extension to Event-B. Event-B is a formal method, that can be used to model safety-, and business-critical systems. The work may be of interest to a section of the Ada community who are interested in applying formal modelling techniques in their development process, and automatically generating Ada code from the model. We describe a streamlined process, where the abstract modelling artefacts map easily to Ada language constructs. Initial modelling takes place at a high level of abstraction. We then use refinement, decomposition, and finally implementation-level annotations, to generate Ada code. We provide a brief introduction to Event-B, before illustrating the new approach using small examples taken from a larger case study.
\end{abstract}

\section{Introduction}

Event-B [1] is a formal method that can be used in the rigorous development of software systems. It may be used in by industry for business-, and safety-critical systems; to increase confidence in the correctness of the system [2]3. In this paper we focus on the domain of multi-tasking, embedded control systems. Our interest is the application of techniques, and provision of tools, for modelling the systems, and generating code from the models. We illustrate the approach using examples from a case study of an embedded Heater Controller, and we use Ada 1995 [4] as the target language. To be able to link Event-B artefacts to programming constructs we have devised an extension to Event-B called Tasking Event-B. Tasking Event-B concepts are directly influenced by Ada constructs. For instance, Ada tasks are modelled by AutoTask machines, and protected objects are modelled by shared machines, in Tasking Event-B.

We continue with section 1.1 in which we discuss our motivation. Section 2 provides a brief introduction to the Event-B approach. Section 3 provides an overview of the Tasking Event-B extension. In Section 4 we present more details of Tasking features and the translation to Ada. Section 5 describes how we can $\mathrm{read} /$ write directly to memory. Section 6 provides an overview of tooling issues, and Section 7 provides a summary and discussion. 


\subsection{Motivation}

The Event-B method, and supporting tools [5], have been developed during the the EU DEPLOY [6] project. A number of the industrial partners, associated with the project, have been interested in the formal development of multi-tasking, embedded control systems. However, automatic generation from Event-B models, for these type of systems, was absent from the approach. We chose Ada as a basis for our approach, not only because of it's suitability for the application domain, but it also serves as a useful reference for our code generation constructs. Ada constructs match well with Event-B modelling elements, and this serves to simplify the translation to code. We do not, however, formally model all aspects of the implementation, e.g. time. We model the behaviour that relates to the control flow specified in the task bodies; for which we provide Event-B semantics. We developed a case study [7] of a Heating Controller to validate the code generation approach. The case study is an analogue of many embedded systems, where inputs from the environment are received and processed, and may have some effect in the environment caused by its outputs.

\section{An Overview of Event-B}

The Event-B method [1] was developed by J.R. Abrial, and uses set-theory, predicate logic and refinement to model discrete systems. The basic structural elements of Event-B models are contexts and machines. Contexts are used to describe the static aspects of a system, using sets and constants; the relationships between them are specified in the axioms clause. Machines are able to see Contexts; the content of a Context is visible and accessible to a machine. Machines are used to describe the dynamic aspects of a system, in the form of state variables, and guarded events, which update state. Safety properties are specified using the invariants clause. The invariants give rise to proof obligations, which are generated automatically by the tool; a large number of the proof obligations may be discharged without user intervention by auto-provers. Where auto-provers fail to discharge proof obligations, the user guides the interactive prover. They proceed by suggesting strategies, and sub-goals in the form of hypotheses, in the endeavour to complete the proof. Refinement is used to show that concrete models satisfy the safety properties of their abstract counterparts.

A fragment of an Event-B specification is shown in Fig. 1. The specification has variables, which are typed in the invariant. Invariants also describe desired safety properties. The event declares two parameters $t m 1$ and $t m 2$. These are typed in the guard clause, following the where keyword. The third guard describes an enabling condition for the event. When the value of avt $<c t t m 2$ the event is enabled, and the updates described in the actions may take place. Actions may contain deterministic or non-deterministic assignments, or do-nothing (skip); but non-deterministic modelling constructs are removed by the time we reach the implementation level specification. In Fig. 1 the action assigns TRUE to the variable $h s c$. 

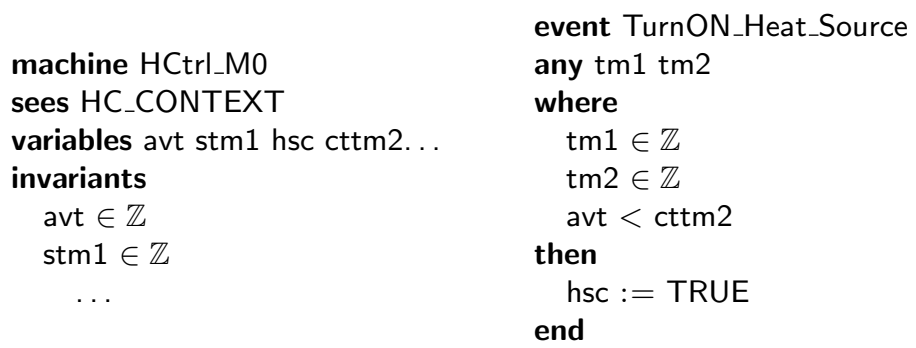

Fig. 1. Example of Textual Event-B

\section{An Overview of Tasking Event-B}

Tasking Event-B is an extension to Event-B, but includes some restrictions to ensure the code is implementable. An Event-B operational semantics underpins the extension. As a means of verifying consistency between tasking Event-B and higher-level abstractions, the Tasking Event-B can be translated to a standard Event-B representation. Then using the Rodin tool we can show that this generated model refines the abstract development.

During the development, before the tasking Event-B stage, we use model decomposition to tackle complexity. The Rodin tool supports different approaches to decomposition; here we use shared event decomposition [8]. In section 4.1 we provide a more detailed picture of how an abstract model is decomposed into its sub-models. This decomposition approach results in a partitioning of the system whereby variables are distributed over decomposed machines. A machine has access to variables of another machine using pairs of synchronized events. Synchronized events allow machines to communicate using parameters; they model atomic access to variables residing in another machine. This synchronization approach is described in more detail in [10. In order to keep track of the synchronizations, the Rodin tool produces a composition component [1] during the decomposition process.

In our approach controllers can be comprised of a number of tasks and, rather than allowing direct communication between controller tasks, we use a shared machine to encapsulate the shared data. This means that synchronizations are taking place between tasking machines and the shared machine. This structure is illustrated in Fig. 2, which describes the relationships between the components of an Event-B development, tasking Event-B and the generated Ada code. In the Tasking Event-B layer, machines are identified as AutoTask, Environ, or Shared. AutoTask Machines model controller tasks in the implementation level, and are implemented using Ada tasks. Shared Machines model encapsulated shared objects, and are implemented by protected objects. Environ Machines model the environment, and are implemented using Ada tasks.

An example of an AutoTask Machine, from our case study [7/12, is shown in Fig. 3. It is a descendant of the fragment shown in Fig. 1, following a number of refinement and decomposition steps. The machine of Fig. 3 is an implementation 


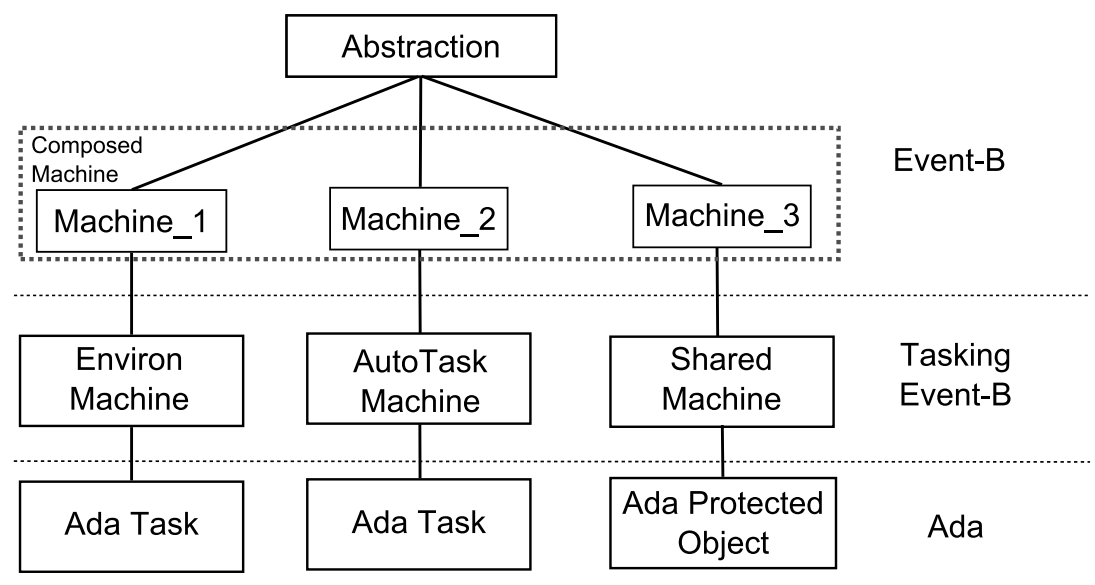

Fig. 2. Heating Controller Artefacts

level refinement, as indicated by the autotask annotation. As a convention, we prefix event names of the environ machine with $\mathbf{E N}$, the shared machine with SO, and temperature controller with TC. We specify some tasking features such as the task type (e.g. periodic, triggered, one-shot, and repeating); the priority, and the task body. A main feature of the tasking level specification is the task body; this is used to specify flow control aspects of the task, with respect to the events that already reside in the machine. The task body may contain clauses, such as sequence, loop, and branch; and uses a programming-style syntax, e.g. ;, do, if and event names. Notice that there is no explicit use of a synchronization construct in the task body, we only refer to events that are local to the tasking machine. The code generator tool uses the composition component, mentioned previously, to find any synchronizations (if they exist) and then generates the implementations. Synchronizations between AutoTasks and the Shared machine are implemented using protected procedures. Synchronizations between AutoTask and Environ machines are implemented as rendezvous, or direct memory access as required. A machine's events can model local (wrt the machine) state updates, subprograms, or branching and looping constructs. As indicated earlier, protected procedure calls are modelled using synchronized events in the task body; this is used when two machines communicate. If an event just updates local state, then updates are mapped to assignment clauses in the target, rather than incurring the overhead of a subprogram call. An Output construct is provided to allow text output to a console during simulation.

In the final, deployable system, inter-task communication can be prohibited. The main driver for this restriction is that we wish to generate safe multi-tasking code which is compliant with the Ravenscar subset of Ada [13. We may relax this restriction, for environment tasks, to simulate the environment. 


\subsection{Event-B Development}

At the top we show the most abstract model of the system where we specify the system's main functionality, such as modelling the increase/decrease of the target temperature, polling of the temperature sensors, calculation of the average temperature, and activation of the heat source and alarms. In the first refinement we introduce sensing and actuation. Sensing events model polling of the state of the increase/decrease buttons, the temperature sensors, and the heater sensor. Actuating events model the updates of target, and current temperature displays. We also model actuation occurring as a result of controller decisions, such as turning the heat on/off, and activating the various alarms. We decompose our model in two stages; we first separate the controller subsystem, the part of the system that should be implemented, from its surrounding environment. In the second stage we decompose the controller subsystem; we identify three controller tasks, and a protected object. The structure of the decomposition is visible in the diagram in Fig. 5. Following decomposition we add an additional refinement, the Tasking Event-B Layer. This refinement layer is used for our implementation level specification. It is necessary to use refinement here, since the automatically generated files (from the decomposition tool) cannot be modified.

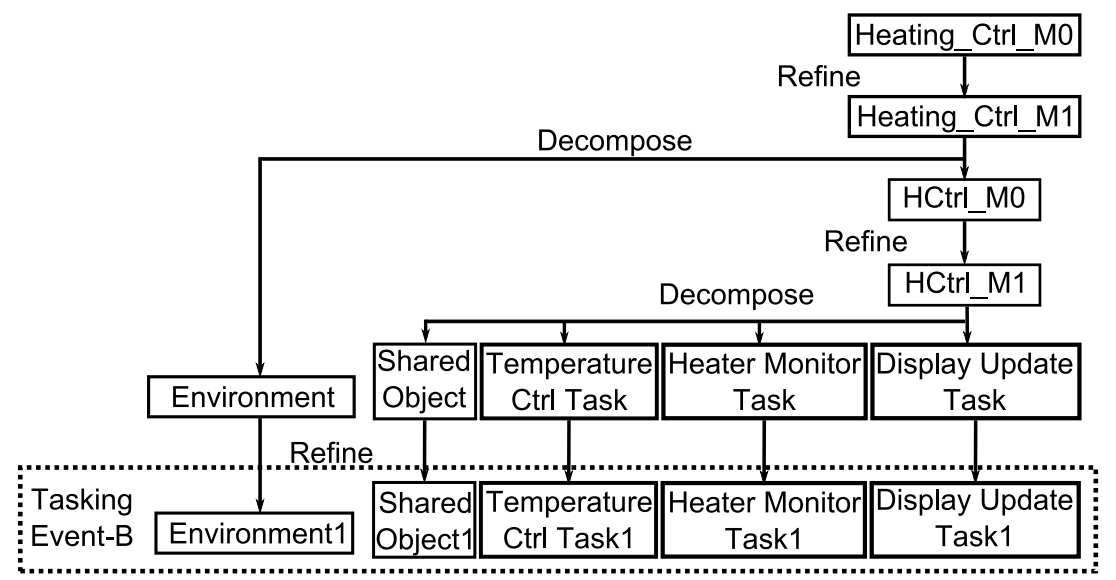

Fig. 5. The Development Approach

\subsection{Guiding Code Generation with Tasking Event-B}

In the previous step we decomposed into five machines; one modelling the environment, one modelling each of the three controller tasks, and one modelling the protected object. We should now add the Tasking Event-B annotations to guide code generation. The first step is to use annotations to identify the machines as being an AutoTask, Environ or Shared machine. With Environ, or 
AutoTask machines, we also add a task body specification. The task body is used to constrain the Event-B model, in such a way that it can be implemented using programming constructs, such as sequence, branch and subprogram calls. The generated code is viewed as an implementation of a schedule of events.

In the discussion that follows, we use the temperature control task event Temp_Ctrl_TaskImpl from Fig. 3, as an example. We describe how we use Tasking Event-B to specify implementation details, that is, how the controller interacts with the environment. The full task body of the Temp_Ctrl_TaskImpl AutoTask is shown in Fig. 6, it includes a brief description of the activities performed. In (1) the temperature controller uses the TCSense_Temperatures event; in (2) the average temperature is calculated, and so on. The comment identifies the synchronizing event, which is presented here for clarity. The task body gives rise to the Ada code show in Fig. 7. We will look at the translated code in more detail, later in the section.

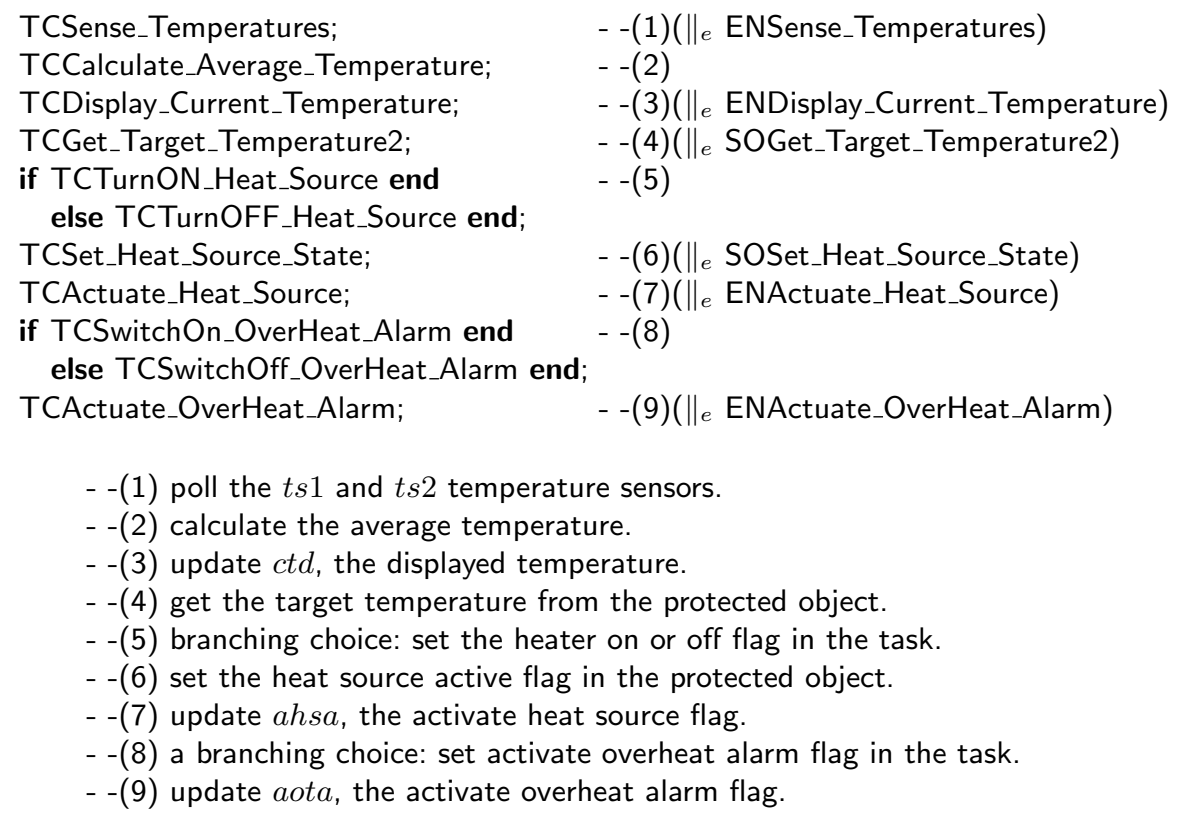

Fig. 6. The Temp_Ctrl_TaskImpl Task Body

The development proceeds by adding annotations to events. In Fig. 8 we see the sensing annotation being used to indicate that an event is used in a sensing role. The sensing keyword is used with both the TCSense_Temperatures and ENSense_Temperatures events. This indicates that the events model polling of the environment; the actuating keyword is similar, except that it indicates that events update values is the environment. Now, returning to the translated 


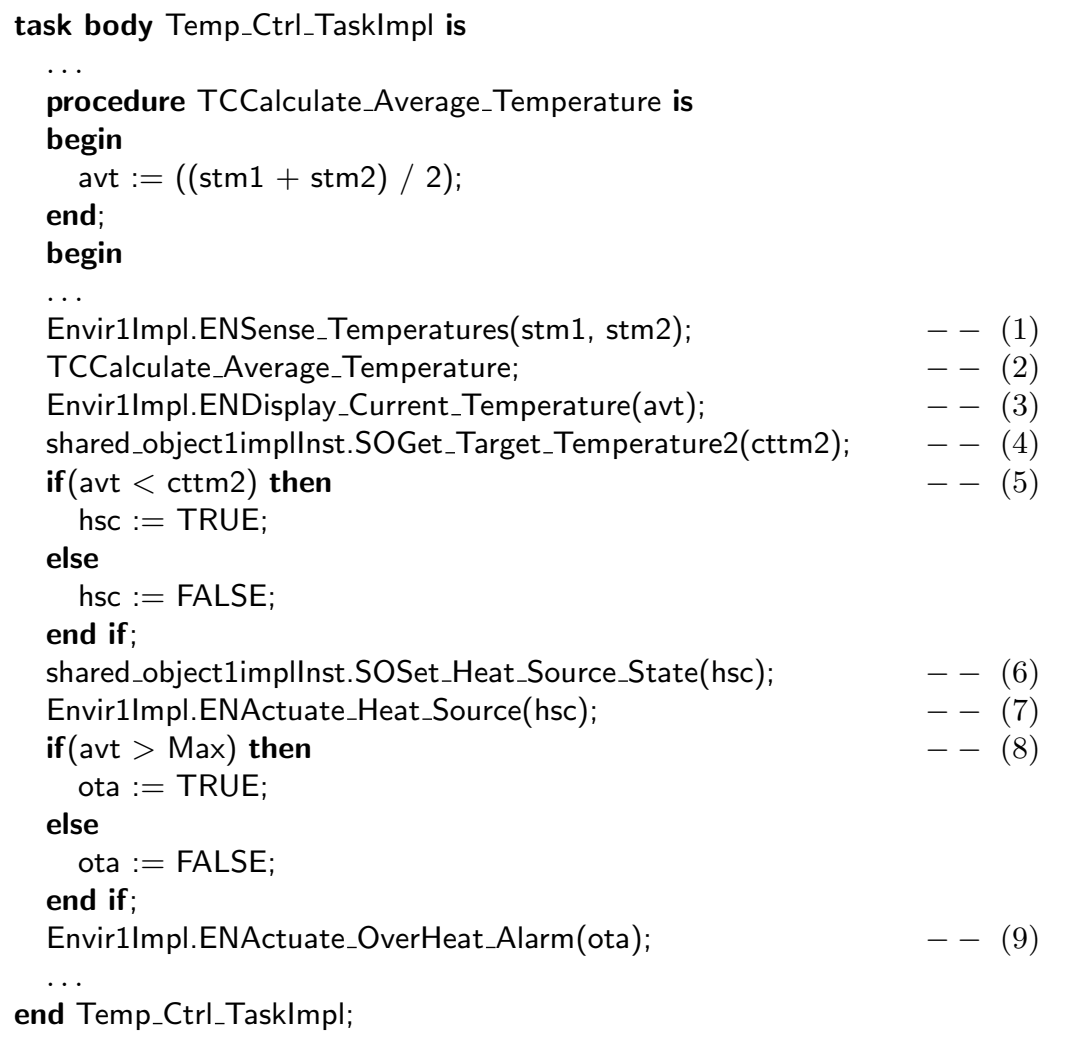

Fig. 7. Implementation of Temp_Ctrl_TaskImpl Task Body
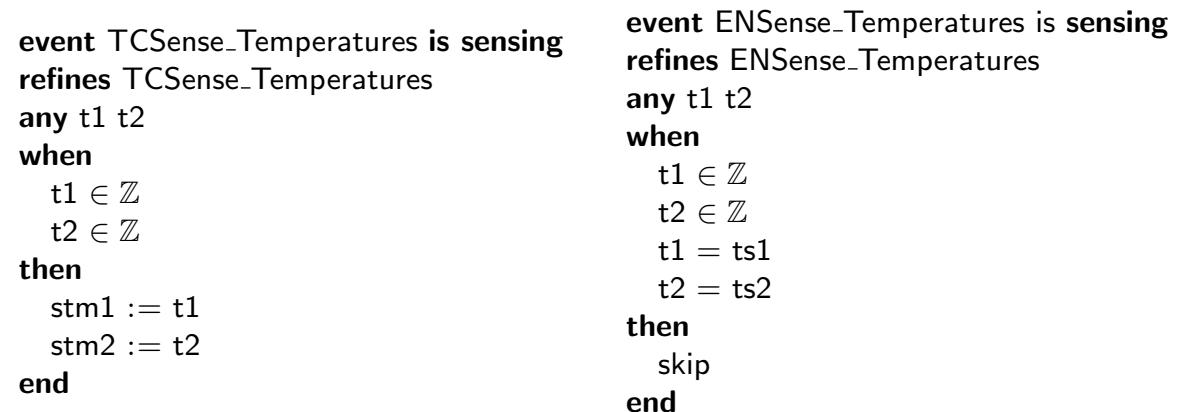

Fig. 8. Synchronization of a Sensing Event 
code, arising from clause (1) of Fig. 6. It results in the following Ada program statement:

Envir1Impl.ENSense_Temperatures(stm1, stm2);

Envir1Impl is the name of the environment task, and ENSense_Temperatures is the name of the task entry. The entry call implements a pair of synchronized events. In the most abstract model (not shown in this paper) stm 1 keeps track of the sensed temperature, ts 1 , using an assignment $s t m 1:=t s 1$. In the decomposition, the two temperature sensing events synchronize to achieve the same result; this is implemented as an entry call. We now describe the relationship between the implementation and the model. The variable stm 1 , appears in the action of the TCSense_Temperatures event, see Fig: 8 , In the translation, the event parameter, $t 1$ is replaced by the variable, stm 1 , and passed as an actual parameter in the entry call. The entry is implemented as an Ada accept statement in the Envir1Impl task, see Fig. 9. The monitored variable $t s 1$ appears in the guard of the ENSense_Temperatures event, of Fig: 8, and translates to an out parameter in the entry signature. Note that in this case the event guard is translated to an assignment in the implementation. When returning from the entry call, the value held by the out parameter is assigned to the actual parameter; that is, $s t m 1:=t 1$, in our example. Since $t 1=t s 1$ we have $s t m 1:=t s 1$, as required.

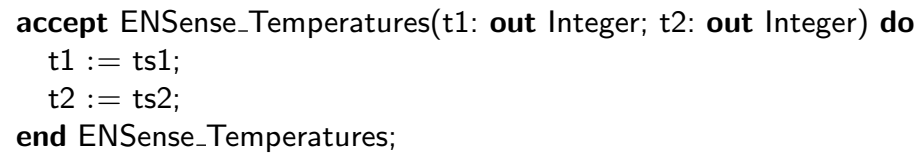

Fig. 9. Implementation of ENSense_Temperatures

The translation of events of shared machines is similar, except that we implement the machines as protected objects with procedures. The translation of synchronized events is otherwise the same, with respect to the mapping of event parameters to subroutine parameters.

\section{$5 \quad$ Writing Directly to Memory Locations}

So far we have described an approach which facilitates interation with the environment using rendezvous. However, we also provide an alternative approach, where the developer specifies some memory locations to read from, and write to. We provide a feature which allows developers to annotate event parameters with address information; using the $a d d r$ keyword. Use of the $a d d r$ address keyword is shown in Fig. 10. We specify a memory location, and its number base. In the example, $t 1$ is given the address ef 14 in base 16 . We can see, on the right of the 
figure, the generated Ada code. The parameter $t 1$ has been mapped to the integer variable declaration $\mathrm{t} 1$ : Integer. The address of the variable has been set using the following statement, the pragma Atomic(t1) statement is used to indicate that any access to $t 1$ must occur atomically. In the TCSense_Temperatures procedure implementation of Fig. 10, the variable $t 1$ appears on the right-hand side of the assignment. When the statement is executed, the value is read from the memory location accessed by $t 1$, and assigned to $s t m 1$. This approach does differ from the entry approach described in the previous section. Entry calls are atomic, whereas we are using non-atomic statements. For this reason the environment must be responsible for ensuring that the implementation of sensing events with multiple read actions, and actuating events with multiple write actions, are performed atomically (we do not envisage mixing sensing and actuating in a single event).
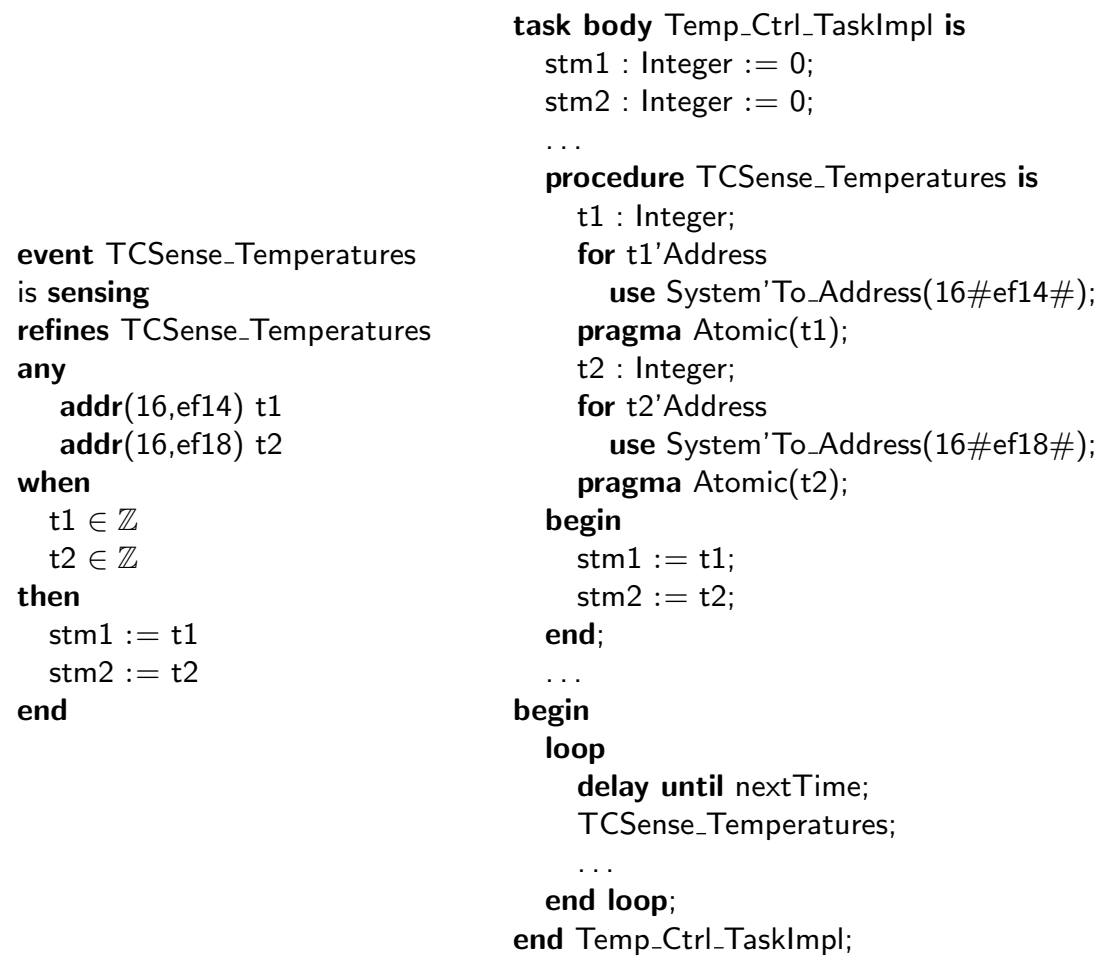

Fig. 10. Addressed Variables: Specification and Implementation

Using a combination of the approaches described in this paper, we can simulate interaction with the environment in the early stages of development, using entry calls.. Later in the development we can choose to read from, and write to, memory directly. To do this we simply add the address information to the rel- 
evant variables. We also have the option of the environment simulation reading from, and writing to memory.

\section{Tooling}

The Rodin tool [5], based on the Eclipse Platform [14, is a complete development environment for Event-B. We have extended the methodology and tools to add implementation level specification, using Tasking Event-B. Tasking Event-B and the code generators are fully integrated into the Rodin toolset, see Fig 11 . When a development is ready for translation to code we have a simple pop-up

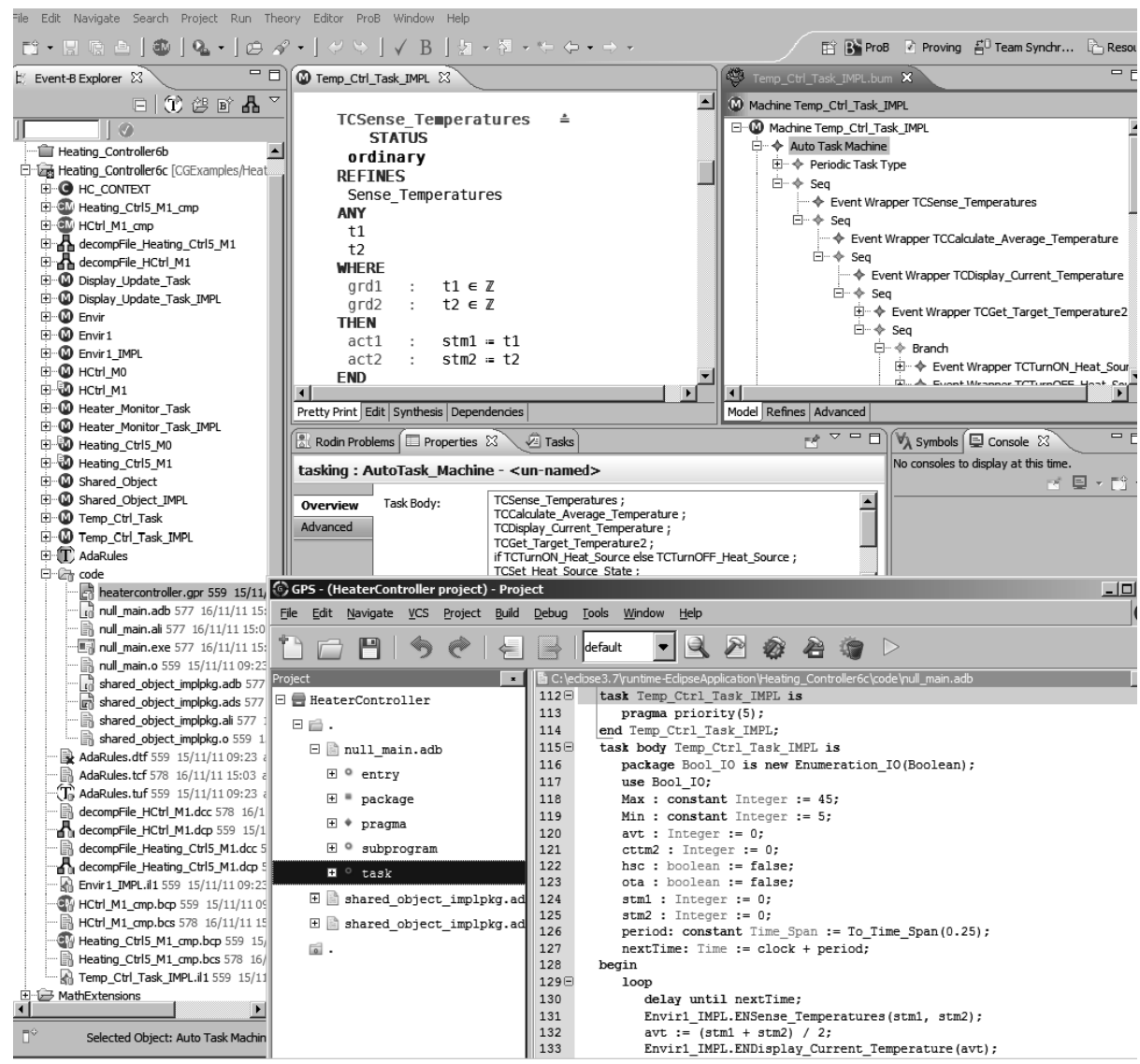

Fig. 11. Code Generation Tools

menu with translation options. The code generators use the Tasking Event-B model, and a two-step process, see Fig. 12 . The first step generates a Common 


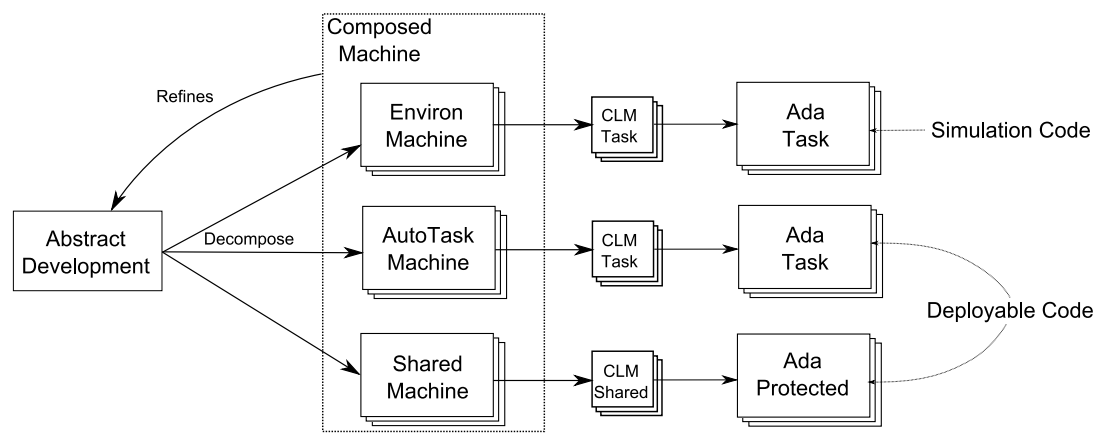

Fig. 12. Two-Step Code Generation

Language Model (CLM); the CLM is an abstraction of commonly used software constructs. The abstract tasks and shared objects of the CLM are then used in the translation to Ada. The Ada translator generates the main procedure file, and specification and body files, in a directory ready for compilation. We have been successfully compiling and executing the generated code, using the GPS tool from AdaCore [15]. The only additional effort has been the creation of the project file; this may also be automated in the future.

\section{Conclusions}

In this paper we have described our methodology and tools for linking EventB, through the use of the Tasking Event-B extension, to Ada code. We relate the Event-B modelling artefacts to their Ada counterparts; and, using the case study, we explain the relationship between the modelling abstraction and implementation in more detail. We have explained how Event-B is augmented with Tasking Event-B annotations, these are used to guide the code generator to produce code. For example, annotations identify the role of the machines in the implementation; a machine may be an AutoTask machine, Environ machine or Shared machine. AutoTask and Environ machines have a task body in which we are able to specify flow of control. This is done through the use of the sequence, branch and loop constructs. We make use of the tool-driven decomposition approach, to structure the development. This allows us to partition the system in a modular fashion, reflecting Ada implementation constructs. Decomposition is also the mechanism for breaking up complex systems to make modelling and proof more tractable. As part of the specification we indicate which of the events take part in sensing and actuating roles; we describe the relationship between event parameters, and their role in the implementation of sensing and actuating events. We extend the sensing and actuating features to allow specification of direct reads from, and writes to memory.

In a wider context, the work we have undertaken is to improve the approach for modelling of, and providing implementations for, multi-tasking embedded 
control systems. In this sense the case study can be seen as representative of the style of interactions, using sensing and actuation, in a domain where controllers are continuously monitoring and reacting to the environment. This work provides a basis for future developments in our sphere of interest, and will continue in the Advance project [16], and others. The Tasking Event-B control flow language has been given Event-B semantics, although we do not formalize every aspect of Tasking Event-B, such as modelling timing, or priority. With regard to modelling time, several projects are under way, investigating timing related issues [17. We can use the Tasking Event-B model to generate an Event-B model of the implementation, using the Event-B semantics. We can show that this model refines the abstract development, thus showing that the properties of the abstract development are satisfied.

\subsection{Related Work}

The closest comparable work is that of Classical-B's code generation approach [18 using B0 [19]. B0 consists of concrete programming constructs, these map to programming constructs in target programming languages. B0 can be translated to Ada, but there is no support for concurrency. Code generation of B to embedded systems was carried out in [20, where the implementation results in sequential code. Some consideration is given, in [21], to the use of an Event-B-like syntax for analysis of multi-tasking programs. By comparison, we use the task body for scheduling, rather than taking a purely interrupt driven approach; we have yet to incorporate modelling of interrupts in Tasking Event-B.

$\mathrm{VDM}++22$ may be used to generate code, it is an object-oriented extension to VDM-SL formal specification language. It has been used to model real-time systems, see 23. The paper describes a controller and environment model similar to our own. They define an abstract operational semantics to describe additional modelling features, whereas we use Event-B semantics. They model time, and asynchronous communication, whereas we do not address these issues in the work presented here. However, the specification of timing properties is of great interest to us; and work has been done to address the issue in Event-B such as [24, or more recently [17. Scade 25] is an industrial tool for formally modelling embedded systems. It provides a graphical approach to specification, and has a certified code generator. It has a similar control flow approach to that of UML-B statemachines [26].

\section{References}

1. Abrial, J.R.: Modeling in Event-B: System and Software Engineering. Cambridge University Press (2010)

2. Russo, A.: Formal Methods in Industry: The State of Practice of Formal Methods in South America and Far East (2009)

3. Metayer, C., Clabaut, M.: Dir 41 case study. 27] 357

4. Taft, T., Tucker, R., Brukardt, R., Ploedereder, E., eds.: Consolidated Ada reference manual: language and standard libraries. Springer-Verlag New York, Inc., New York, NY, USA (2002) 
5. RODIN Project. (at http://rodin.cs.ncl.ac.uk)

6. The DEPLOY Project Team: Project Website. (at http://www.deployproject.eu/)

7. Edmunds, A., Rezazedah, A.: Event-B Wiki: Development of a Heating Controller System. (at http://wiki.eventb.org/index.php/Development_of_a_Heating_Controller_System)

8. Butler, M.: Decomposition Structures for Event-B. In: Integrated Formal Methods iFM2009, Springer, LNCS 5423. Volume LNCS., Springer (2009)

9. Silva, R., Pascal, C., Hoang, T., Butler, M.: Decomposition Tool for Event-B. Software: Practice and Experience (2010)

10. Edmunds, A., Butler, M.: Tasking Event-B: An Extension to Event-B for Generating Concurrent Code. In: PLACES 2011. (2011)

11. Silva, R.: Towards the Composition of Specifications in Event-B. In: B 2011. (2011)

12. Edmunds, A., Rezazedah, A.: Event-B Project Archives: Tasking Event-B Tutorial. University of Southampton. (at http://deploy-eprints.ecs.soton.ac.uk/304/)

13. Burns, A., Dobbing, B., Vardanega, T.: Guide for the use of the Ada Ravenscar Profile in high integrity systems. Ada Lett. XXIV (2004) 1-74

14. The Eclipse Project: Eclipse - an Open Development Platform. (Available at http://www.eclipse.org/)

15. AdaCore: GNAT Programming Studio. (Available at http://www.adacore.com/home/)

16. The Advance Project Team: The Advance Project. (Available at http://www.advance-ict.eu)

17. Sarshogh, M., Butler, M.: Specification and Refinement of Discrete Timing Properties in Event-B. In: AVoCS 2011. (2011)

18. Abrial, J.: The B Book - Assigning Programs to Meanings. Cambridge University Press (1996)

19. ClearSy System Engineering: The B Language Reference Manual. (Version 4.6 edn.)

20. Bert, D., Boulmé, S., Potet, M., Requet, A., Voisin, L.: Adaptable Translator of B Specifications to Embedded C Programs. In Araki, K., Gnesi, S., Mandrioli, D., eds.: FME. Volume 2805 of Lecture Notes in Computer Science., Springer (2003) 94-113

21. Stoddart, W., Cansell, D., Zeyda, F.: Modelling and Proof Analysis of Interrupt Driven Scheduling. In Julliand, J., Kouchnarenko, O., eds.: B. Volume 4355 of Lecture Notes in Computer Science., Springer (2007) 155-170

22. CSK Systems Corporation: (The VDM++ Language Manual)

23. Verhoef, M., Larsen, P.G., Hooman, J.: Modeling and Validating Distributed Embedded Real-Time Systems with VDM++. In Misra, J., Nipkow, T., Sekerinski, E., eds.: FM. Volume 4085 of Lecture Notes in Computer Science., Springer (2006) $147-162$

24. Degerlund, F., Grnblom, R., Sere, K.: Code Generation and Scheduling of Event-B Models (2011)

25. Berry, G.: Synchronous Design and Verification of Critical Embedded Systems Using SCADE and Esterel. In Leue, S., Merino, P., eds.: FMICS. Volume 4916 of Lecture Notes in Computer Science., Springer (2007) 2

26. Snook, C., Butler, M.: UML-B: A Plug-in for the Event-B Tool Set. 27] 344

27. Börger, E., Butler, M.J., Bowen, J.P., Boca, P., eds.: Abstract State Machines, B and Z, First International Conference, ABZ 2008, London, UK, September 16-18, 2008. Proceedings. In Börger, E., Butler, M.J., Bowen, J.P., Boca, P., eds.: ABZ. Volume 5238 of Lecture Notes in Computer Science., Springer (2008) 